\title{
Vibration Analysis of a Suspension System Subject to High Level of Measurement Noise
}

\author{
Ferenc Szauter and György Istenes \\ Széchenyi István University \\ Research Center of Vehicle Industry \\ Győr, Hungary \\ Email: szauter@sze.hu, istenes.gyorgy@sze.hu
}

\author{
Gábor Rödönyi \\ Systems and Control Laboratory, \\ Computer and Automation Research Institute of \\ Hungarian Academy of Sciences \\ Email: rodonyi.gabor@sztaki.mta.hu
}

\begin{abstract}
Using only vertical acceleration measurements for the sprung and unsprung masses of a suspension system of a commercial city bus, the goal of the paper is to develop an analysis method to find the vibration modes of the mechanical system from data measured during real life operation. The identified vibration modes can be used to (in)validate first principle physical models of the system, while the identified ARMA models can be used to develop uncertainty models. The challenge in the problem is that the measurements are subject to very high level of noise due to maneuvering of the vehicle, nonlinear effects of the suspension system, vibration of the engine and the gear system, and sensor noise. Nonparametric and parametric modeling methods are applied to evaluate the quality of the measurements and find the invariant properties of the suspension system. It is shown based on multiple experiments that independently of the actual road properties and operating conditions, eigen-frequencies of some vibration modes can be determined with relatively small uncertainty, while the corresponding damping factors have varying amount of uncertainty. Comparing the results with the modes of a full car vehicle model developed based on physical considerations, it can be concluded that an identification algorithm for obtaining the parameters of the physical model must be complemented with a suitable uncertainty modeling and classification.
\end{abstract}

\section{INTRODUCTION}

Assessing technical conditions of the road network and of fleets of public vehicles in a city provides an important support to operators in making decisions on the scheduling and harmonization of maintenance works. The long range goal of our project is to equip a fleet of buses with relatively low cost hardware to collect data about vertical vibrations and to develop a tool chain for evaluating both road conditions and technical state of the vehicles. The resulted system is to operate during normal every-day operation continuously monitoring the vehicles and supporting decision making. Toward the construction of such a monitoring system, the following tasks have to be performed.

Construction of a mathematical model for describing nominal vibration mechanism. This model will serve as a reference for evaluating both the technical condition of the suspension system of a specific vehicle, and conditions of road surface. The reference model should be constructed based on experiments of specific circumstances where the road surface is known and undamaged, the road is straight, the vehicle speed is constant, the vertical dynamics of the vehicle is excited by well specified test obstacles placed on the road. With repeated experiments a set of nominal models are obtained from which a so called nominal uncertainty model set can be constructed, [3], [6].

Furnished with the nominal model set, measurements are collected under specific test conditions, like different road surfaces and vehicle suspension characteristic. Uncertainty modeling is repeated to obtain a set of uncertainty models. We expect that by using a sufficient nominal model, the effects of a normal vehicle behavior can be filtered out of the data, and the uncertainty models can be applied to classify anomalous effects due to bad road or vehicle conditions.

To start any identification process we need data. Experiments are expensive and time consuming, therefore sometimes we have to make the best of measurements collected during normal every-day operation of the system. In these circumstances the data of commercial city buses contain the effects of several issues: road surface variations, dynamics of the suspension system excited by the disturbing lateral and longitudinal accelerations, roll and pitch motions of the vehicle, variations in the mass of load, and sensor noise. Only a small part of the information is due to the vertical suspension system and the vertical road excitation. We say that these measurements are subject to very high level of noise. It is important to know what information can be obtained from the data like this. To this end, we seek in this paper the "invariant factors", that is, parameters that can be gained independently of road and operation conditions.

Preliminary results can be found in [10] and [11], where nonparametric and parametric (AR, ARMA) spectral analysis were applied to evaluate vibration modes in terms of ride comfort and invalidate the physics based low order "quarter car" model, respectively.

In this paper it is shown that by identifying a sequence of autoregressive moving average (ARMA) models, some disturbing effects can be identified, and some invariant parameters can be estimated which can be useful in the validation and identification process of a 7-degree of freedom, first principle linear suspension model, the so called "full car" model. The comparison of the invariant parameters obtained from the ARMA models and those of the full car model suggests that a sufficiently detailed physical model endowed with uncertainty models can be identified even in the presence of high level of noise.

The paper is organized as follows. In Sections II and III the experimental conditions and the quality of measurements are 


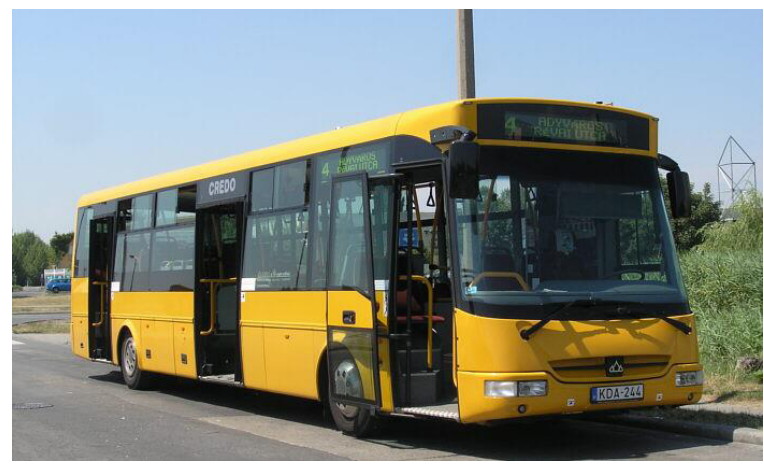

Fig. 1. Test vehicle

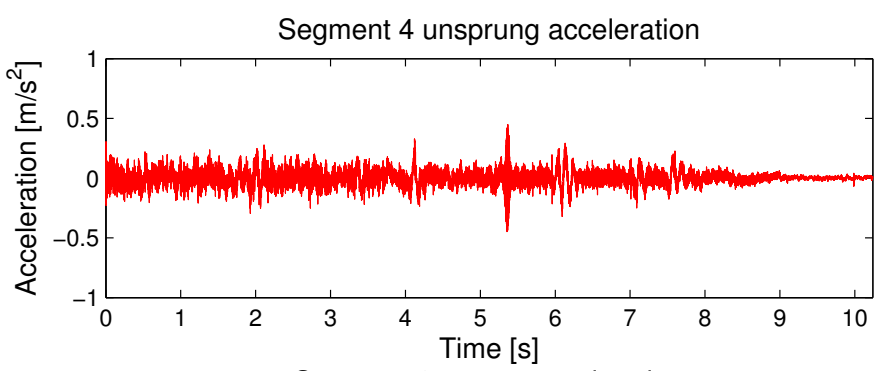

Segment 4 sprung acceleration

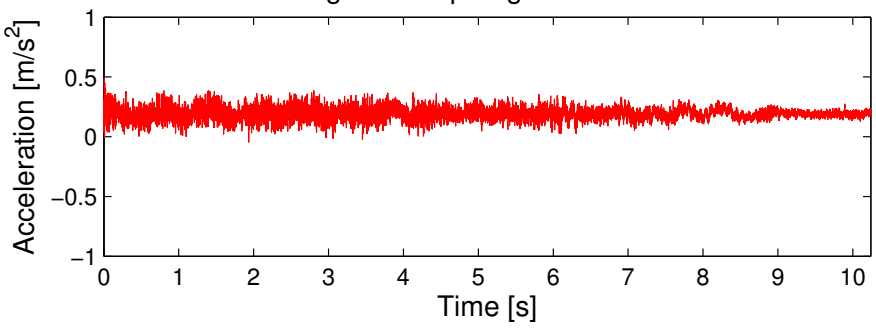

Fig. 2. Measured time-domain acceleration data at the front right. Unsprung mass (top) and sprung mass (down).

discussed. The invariant parameters of the suspension system under the time-varying uncertain effects are determined in Section IV.

\section{EXPERIMENTAL CONDITIONS AND PREPROCESSING}

The test vehicle shown in Fig. 1 was equipped with seven accelerometers. Near the four spindle on each of the Zaxis direction PCB ICP-type accelerometers were mounted to measure the vertical acceleration of the unsprung masses. On the floor plate (except for the driver side), over these sensors, differential DC MEMS accelerometers were mounted along the $\mathrm{Z}$-axis direction to measure the vertical acceleration of the sprung mass. In addition to the acceleration transducer system a GPS based track recorder was also installed in order to support data segmentation.

The measurements for the analysis in this paper were collected on the normal route-way of the city bus in real traffic conditions. The data was segmented into smaller records. Eight pieces, each of length $10.24 \mathrm{sec}$, were cut. The measurements are affected by both the road surface and the vehicle dynamics. A sample data record can be observed in Figure 2.

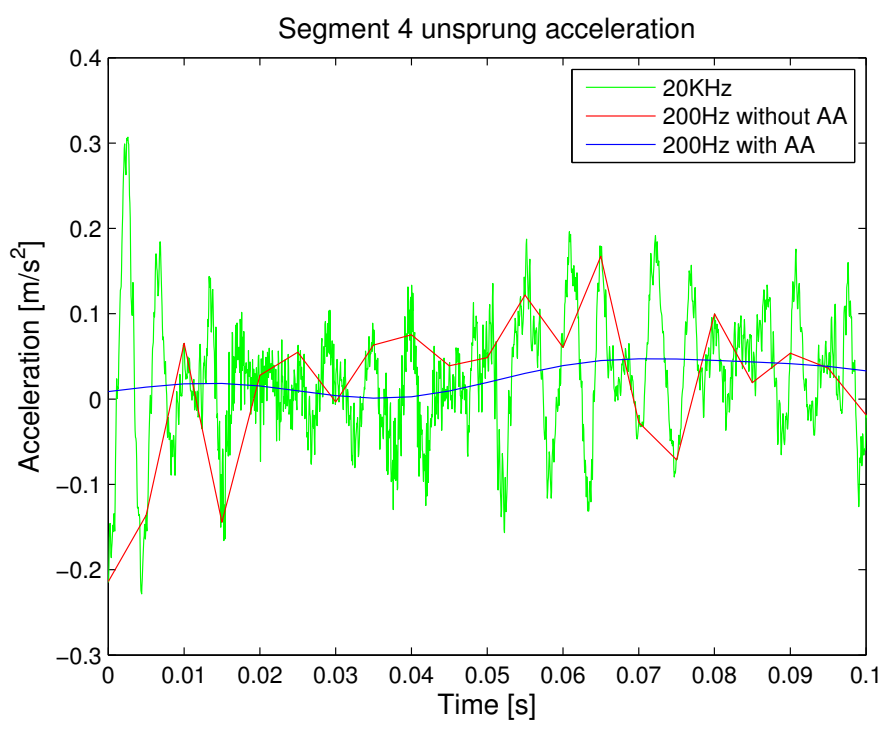

Fig. 3. Anti-aliasing filtering

The row data was sampled by $20 \mathrm{kHz}$ resulting in a large data set. In order to reduce the amount of data, a new sampling frequency was chosen to be $f_{s}=1 / T_{s}=200 \mathrm{~Hz}$ which is sufficiently high to capture the dynamics of the suspension system. However, resampling must be taken with care, due to aliasing effects, as demonstrated in the following.

An ideal filter (rectangular window) was used as antialiasing filter. First, the DFT components of the $20 \mathrm{kHz}$ signal beyond $25 \mathrm{~Hz}$ were zeroed out. Then the filtered $20 \mathrm{kHz}$ signal was reconstructed by using inverse DFT. This filtered signal can be resampled with $f_{s}=200 \mathrm{~Hz}$. The resulted data set consisted $N=2048$ samples.

In Figure 3 a $0.1 \mathrm{sec}$ period of the original signal and the filtered and resampled signal are plotted with green and blue lines, respectively. For illustration of the necessity of antialiasing filtering, the original signal is resampled also without filtering. The result is plotted by a red line in Figure 3. The difference between the two resampled signals can be observed also in the frequency domain in Figure 4. The spectrum of the filtered and resampled signal coincides with the spectrum of the original $20 \mathrm{kHz}$ signal. Without antialiasing filtering (red line), high frequency components of the signal (noise) appear at lower frequencies after resampling and alter the low frequency components of the signal. For more details on aliasing effects, see [2].

\section{SPECTRAL ANALYSIS}

In order to have a first impression on the information the data may contain, a preliminary spectral analysis can be performed. Both non-parametric and parametric methods can be considered. In this paper, the parametric ARMA method is used not only for spectral analysis, but also for determining the possible modes of the suspension system. The approach we follow is to separate invariant factors (possibly due to suspension system dynamics) from uncertainties that may vary from experiment to experiment. In this section the applied mathematical tools are summarized. 


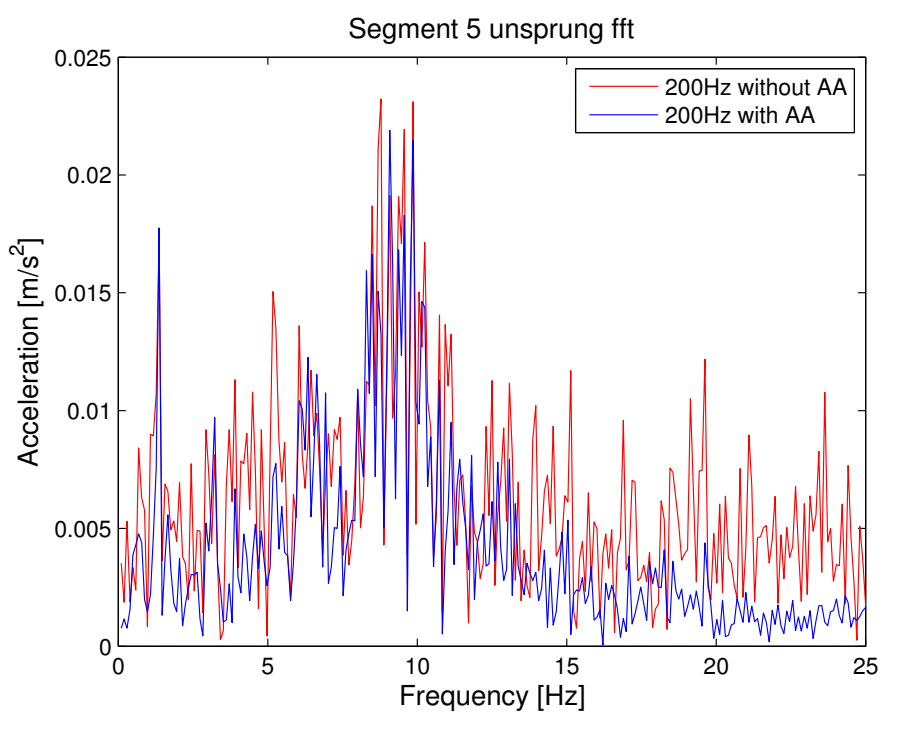

Fig. 4. Effects of anti-aliasing filtering on the low frequency components of the signal.

The goal of spectral estimation is to describe the frequencydistribution of the power contained in a signal, based on a finite set of data. The power spectral density (PSD) of a wide-sense stationary random process $x_{t}$ is mathematically related to the autocorrelation sequence by the discrete Fourier transformation (DFT). In terms of normalized frequency, this is given by $P_{x x}(f)=T_{s} \sum_{m=-\infty}^{\infty} R_{x x}(m) e^{-i 2 \pi m f T_{s}}$, where the autocorrelation sequence is defined by $R_{x x}(m)=$ $\frac{E\left[\left(x_{t}-\mu\right)\left(x_{t+m}-\mu\right)\right]}{\sigma^{2}}$, where $\mu$ denotes the mean of the sequence, $\sigma^{2}=\int_{-f_{N}}^{f_{N}^{2}} P_{x x}(f) d f$ denotes variance with Nyquist frequency $f_{N}=\frac{1}{2 T_{s}}$, and $E($.$) denotes expectation, see [5], [7].$

\section{A. Nonparametric methods}

The PSD can be estimated directly from the sampled data (nonparametric methods). The simplest method is the periodogram which is the Fourier transform of the biased estimate of the autocorrelation sequence

$$
\hat{P}_{x x}(f)=\frac{T_{s}}{N}\left|\sum_{t=1}^{N} x_{t} e^{-i 2 \pi f(t-1)}\right|^{2}, \quad-f_{N}<f<f_{N}
$$

For a one-sided periodogram, the values at all frequencies except 0 and $f_{N}$ are multiplied by 2 so that the total power is conserved. Periodograms can be computed by using DFT.

The periodogram is asymptotically unbiased, however, in some cases the periodogram is a poor estimator of the PSD even when the data record is long. This is due to the variance of the periodogram,

$$
\operatorname{Var}\left(P_{x x}\left(f_{k}\right)\right)= \begin{cases}2 P_{x x}^{2}\left(f_{k}\right), & k=0 \text { or } k=\frac{N}{2} \\ P_{x x}^{2}\left(f_{k}\right), & k=1,2, \ldots, \frac{N}{2}-1\end{cases}
$$

which does not tend to zero as the data length $N$ tends to infinity. Here, $P_{x x}\left(f_{k}\right)$ denotes the periodogram defined by (1) computed by DFT. In statistical terms, the periodogram is not a consistent estimator of the true power spectral density of a wide-sense stationary process.
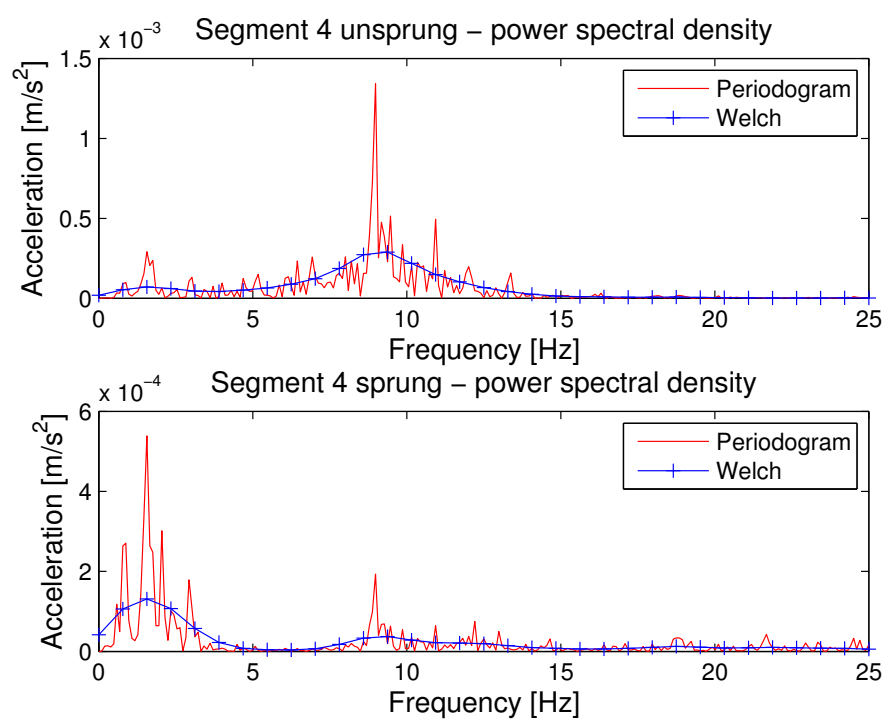

Fig. 5. Power spectral density of the measurements of Segment 4

An alternative method is by Welch, [12]. It breaks the time series into segments to reduce the variance of the periodogram. Welch's method computes a modified periodogram for each segment and then averages these estimates to produce the estimate of the power spectral density. Welch's method yields a biased estimator of the PSD, however it is asymptotically unbiased. For a fixed length data record, the bias of Welch's estimate is larger than that of the periodogram because the length of the segments is less than the length of the entire data sample. The variance of Welch's estimator is difficult to compute. Basically, the variance is inversely proportional to the number of segments whose modified periodograms are being averaged.

A typical PSD computation is shown in Figure 5, where the periodogram and the Welch's method were used. It can be seen that the variance of the periodogram is quite large. The variance of Welch's method is much smaller, it actually depends on the chosen window length and the overlapping rate.

From Fig. 5, two main resonant peaks of the PSD can be observed. The one is about $1 \mathrm{~Hz}$, the other is about $9 \mathrm{~Hz}$. Based on the physical model of a quarter car suspension model, the two peaks should correspond to the two main modes of the suspension system. In [10] the suspension system is evaluated in terms of ride comfort based on the vibration levels and damping factors computed from the two PSD peaks of the two acceleration measurements and multiple data segments.

In order to further examine the information that can be gained from the data, parametric methods are involved.

\section{B. Parametric methods}

Parametric methods estimate PSD from a signal that is assumed to be output of a linear system driven by white noise. Examples are the Yule-Walker and the Burg method. These methods estimate the PSD by first estimating the parameters of the linear system that hypothetically generates the signal. They 
tend to produce better results than classical nonparametric methods when the data length of the available signal is relatively short. Parametric methods also produce smoother estimates of the PSD than nonparametric methods, but are subject to error from model misspecification.

The model structure applied in this paper is the auto regressive moving average (ARMA) process

$$
\begin{aligned}
& \operatorname{ARMA}(\mathrm{p}, \mathrm{q}): \\
& y_{t}+a_{1} y_{t-1}+\ldots+a_{p} y_{t-p}=e_{t}+c_{1} e_{t-1}+\ldots+c_{q} e_{t-q} .
\end{aligned}
$$

with $e_{t}$ being a white noise process of variance $\lambda$. With given $n$ and $p$, ARMA model parameters are estimated in this paper by iteratively minimizing a quadratic prediction error criterion [5]. With the choice $q=p$, the model will be denoted a single argument by $\operatorname{ARMA}(\mathrm{p})$.

Suppose the model structure ARMA(p) and the model parameters collected in vector $\theta$

$$
\theta=\left[a_{1}, a_{2}, \ldots, a_{p}, c_{1}, c_{2}, \ldots, c_{p}\right]^{T}
$$

are chosen, and the noise variance $\lambda$ are given, the estimated PSD of the output can be expressed by

$$
\Phi_{y y}(f)=|W(f)|^{2} \lambda
$$

as a function of frequency, $f \in\left\{-\frac{f_{s}}{2},-\frac{f_{s}}{2}+\frac{f_{s}}{N},-\frac{f_{s}}{2}+\right.$ $\left.\frac{2 f_{s}}{N}, \ldots, \frac{f_{s}}{2}\right\}$, where

$$
W(f)=\frac{1+\sum_{k=1}^{p} c_{k} e^{-j 2 \pi f k}}{1+\sum_{k=1}^{n} a_{k} e^{-j 2 \pi f k}}
$$

is the transfer function of the model.

For a given data set, the goal of the ARMA model identification is to find the model parameters in vector $\theta$ which minimize the variance $\lambda$ of the prediction error $\varepsilon_{t}(\theta)=y_{t}-\hat{y}_{t}(\theta)$. This problem is equivalent to minimizing the variance of the PSD estimate. For ARMA model parameter identification an iterative search algorithm is used that minimizes a robustified quadratic prediction error criterion [8], [5, Chapter 10.2].

Figures 6 and 7 present the PSD estimate based on identified ARMA(10) and ARMA(20) models, respectively. It can be seen that the higher order model captures better the two modes of interest. From the poles of the ARMA models, the stable complex poles with positive real part are selected, and their associated frequency is computed by

$$
f_{i}=\frac{\left|\ln \left(z_{i}\right)\right|}{2 \pi T_{s}}
$$

where $z_{i}$ denotes a complex pole of the discrete time model and $T_{s}=1 / f_{s}$ is the sampling time. Actually, $f_{i}$ is the frequency of the step response equivalent (zero order hold equivalent) continuous-time resonant mode. Vertical black dotted lines show the frequencies of these lightly damped modes. It can be concluded that high order ARMA models are able to provide good PSD estimates. Concerning the frequencies of the resonant modes, Fig. 8 justify the above statement. It can be seen that by increasing the model order the frequency of the modes in the low frequency range, $[0 \mathrm{~Hz}, 15 \mathrm{~Hz}]$, appear consistently. It can be said that the some frequencies of the modes are invariant against the model order, when the order is sufficiently high to capture uncertainties.

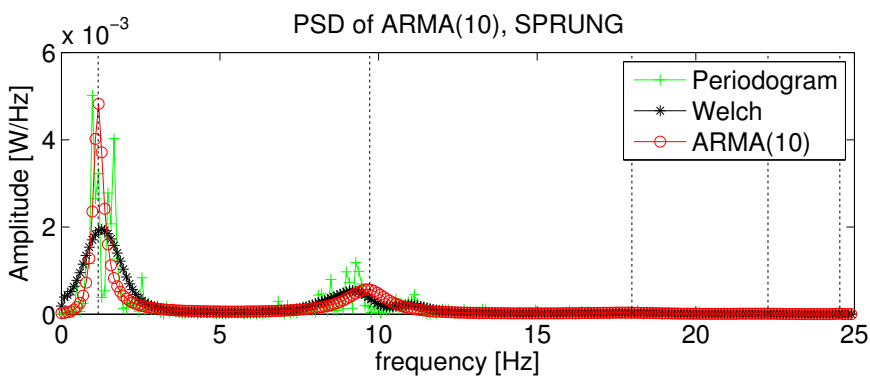

Fig. 6. Power spectrum of ARMA(10) model. Data: segment 1, sprung mass acceleration. Vertical black dotted lines show the frequencies of the lightly damped modes.

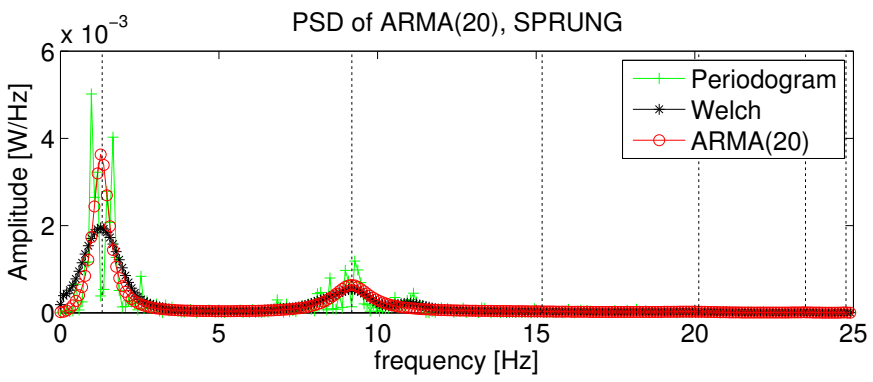

Fig. 7. Power spectrum of ARMA(20) model. Data: segment 1, sprung mass acceleration. Vertical black dotted lines show the frequencies of the lightly damped modes.

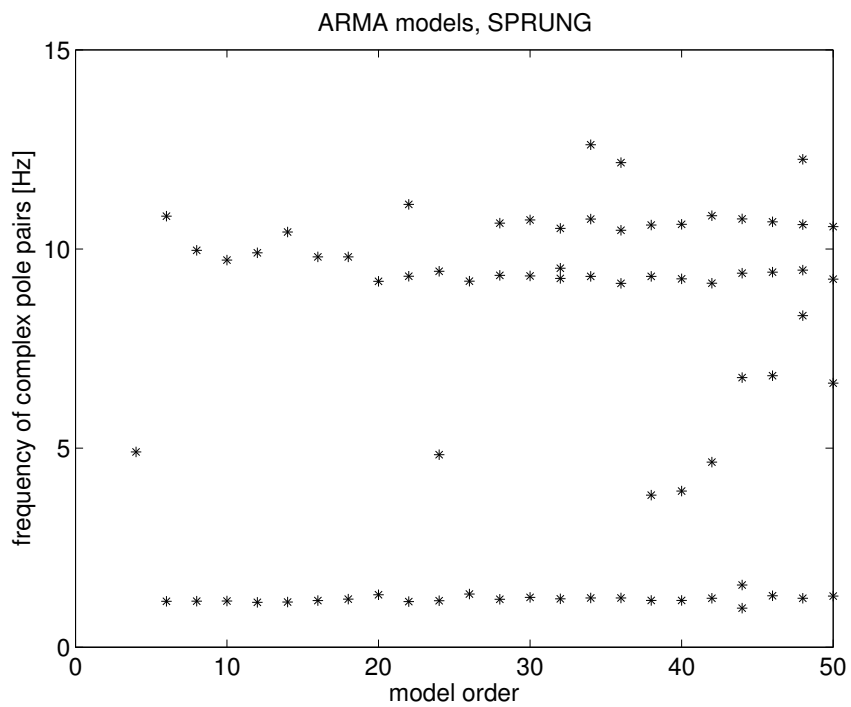

Fig. 8. Frequencies of the identified resonant modes in the different ARMA models.

Beyond spectral analysis, the identification of ARMA models are useful also to determine poles of the linearized model of the suspension system and point at some possible causes of some uncertainties. This is discussed in the next section.

\section{INVARIANT PARAMETERS OF SUSPENSION SYSTEM}

We have seen in Fig. 8 that some frequencies are invariant (do not vary too much) under increasing the model order. The 

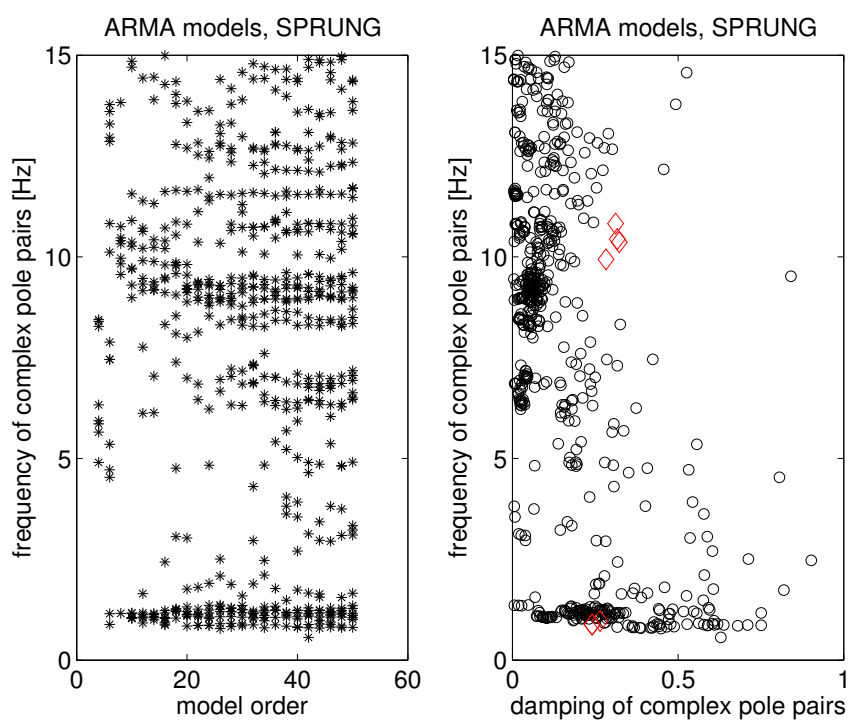

Fig. 9. Frequency and damping of resonant modes in the different ARMA models, sprung mass acceleration, and poles of a full car suspension model.

variation of this invariant frequency parameter increases when other experiments are considered. In Figs. 9 and 10 all 8 road segments are used to generate ARMA models, and all resonant frequencies are plotted versus model order (left) and versus the corresponding damping parameter.

The ARMA models for the unsprung mass acceleration show invariance of frequencies only at $1 \mathrm{~Hz}$ and $4 \mathrm{~Hz}$, the higher modes are strongly influenced by the noise and the uncertainty. In contrast, models for the sprung mass acceleration tell much more, see Fig. 9. Poles at about $1 \mathrm{~Hz}, 7 \mathrm{~Hz}, 9 \mathrm{~Hz}, 11 \mathrm{~Hz}$ and $12 \mathrm{~Hz}$ are much more characteristic, than other frequencies. The value and variance of the corresponding damping parameters plotted on the right hand side show the character of some modes. To be more specific, very small damping, around 1e3 refer to the presence of some periodic disturbance of $7 \mathrm{~Hz}$, $11 \mathrm{~Hz}$ and $12 \mathrm{~Hz}$.

The obtained poles of the ARMA models are compared to the poles of a first principle physical model, the so called full car suspension model. In that model, it is assumed that the vehicle body is rigid, and placed on four independent suspension systems. Through the rigid body, these subsystems are connected. Detailed mathematical model is omitted here, but can be found in references [13] and [14]. The poles of this linear full car suspension model are parameterized in terms of frequency and damping, and denoted in Figs. 9 and 10 by red diamonds. The poles at $1 \mathrm{~Hz}$ are coincide with poles of many ARMA models. Poles at $10 \mathrm{~Hz}$ have a bit larger damping than those of ARMA models. This result enables some hope to construct and identify physically parameterized suspension models, possibly by extending the dynamics by flexible body modes. In every case, the identification must be carried out by joint uncertainty modeling.

\section{CONClusion}

The suspension system of a city bus was analyzed based on the spectral analysis of real acceleration measurements collected in real traffic conditions. Nonparametric and parametric
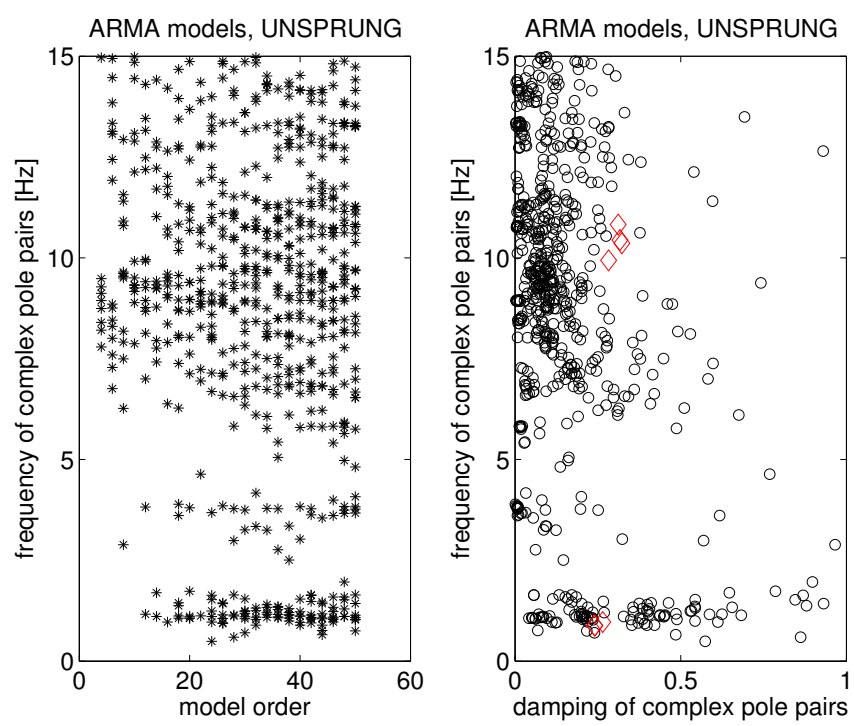

Fig. 10. Frequency and damping of resonant modes in the different ARMA models, unsprung mass acceleration, and poles of a full car suspension model.

tools were applied to qualify the amount of uncertainty in the data. We found that no low order, first principle models (quarter car suspension models) are able to describe the data, specially the data of multiple experiments. The uncertainty due to time-varying effects, neglected dynamics and disturbances, is very large in the data. In this paper we pursued invariant parameters, that can be determined even under varying conditions, multiple experiments, and which were independent on the model order selection.

It can be concluded that some low frequency lightly damped modes are invariant, specially the frequency parameter of these modes. In all experiment and high order ARMA models, the same periodic components are present, so these components can be filtered out for further identification problems. The invariant modes were also compared with the poles of a medium order full car suspension model, and we found close relation between them, which show a direction for further identification work.

Future work includes the identification of higher order physical suspension models with joint identification of black box uncertainty models.

\section{REFERENCES}

[1] J. Bokor, L. Palkovics, P. Michelberger and P. Várlaki, Design of active wheel suspension system using RLQR and $\mathcal{H}_{\infty}$ control, Vehicle System Dynamics, 1993

[2] W.A. Gardner, Statistical spectral analysis. A nonprobabilistic theory, Prentice Hall, Englewood Cliffs, New Jersey

[3] P. Gáspár, Z. Szabó and J. Bokor, Parameter identification of a suspension system and road disturbance estimation. International Journal of Vehicle Systems Modelling and Testing 2:(2) pp. 128-137, 2007

[4] M.H. Hayes, Statistical Digital Signal Processing and Modeling, John Wiley \& Sons, 1996

[5] L. Ljung, System Identification (2nd Ed.): Theory for the User, Prentice Hall PTR, Upper Saddle River, NJ, USA, 1999

[6] P. Michelberger, J. Bokor, A. Keresztes and P. Várlaki, Identification of a Multivariable Linear-model for Road Vehicle (bus) Dynamics From Test Data, International Journal of Vehicle Design 8:(1) pp. 96-114, 1987 
[7] Signal Processing Toolbox User's Guide, The MathWorks, Inc., 2001

[8] System Identification Toolbox User's Guide, The MathWorks, Inc., 2014

[9] D.B. Percival and A. T. Walden, Spectral Analysis for Physical Applications: Multitaper and Conventional Univariate Techniques. Cambridge, UK: Cambridge University Press, 1993.

[10] F. Szauter, Gy. Istenes and G. Rödönyi, Spectral Analysis of Suspension System of a Commercial City Bus, IEEE 14th International Symposium on Intelligent Systems and Informatics, SISY 2016, Subotica, Serbia, August 29-31, pp. 67-72, 2016.

[11] F. Szauter, Gy. Istenes and G. Rödönyi, AR and ARMA Spectral Analysis of Suspension System of a Commercial City Bus, IEEE 17th International Symposium on Computational Intelligence and Informatics, CINTI 2016, Budapest, Hungary, November 17-19, 2016.

[12] P.D. Welch, The Use of Fast Fourier Transform for the Estimation of Power Spectra: A Method Based on Time Averaging Over Short, Modified Periodograms, IEEE Transactions on Audio and Electroacoustics, Vol. AU-15. Pgs. 70-73. 1967

[13] Ferenc Szauter, Tams Pter, Istvn Lakatos, Examinations of complex traffic dynamic systems and new analysis, modeling and simulation of electrical vehicular systems. Mechatronic and Embedded Systems and Applications (MESA), 2014 IEEE/ASME 10th International Conference Senigallia, pp 1-5 Sept. 10-12, 2014 DOI: 10.1109/MESA.2014.6935613

[14] Szauter Ferenc, Pter Tams and Bokor Jzsef, Complex Analysis of the Dynamic Effects of Car Population Along the Trajectories, ASME 2015 International Design Engineering. Technical Conferences and Computers and Information in Engineering Conference. Massachusetts, USA, August 2?5, 2015 Paper No. DETC2015-47075, pp. V009T07A070; 6 pages DOI:10.1115/DETC2015-47075 